\title{
Research on the Integrated Design of Navigation and Guidance Based on DSP+FPGA
}

\author{
Shigang Fan $^{1, a}$ \\ ${ }^{1,2,3}$ High-tech Institute, Fan Gong-ting South Street on the 12th, QingZhou 262500 , China.
}

Keywords: DSP; FPGA; Integration of navigation and guidance; Design research

\begin{abstract}
The master-slave multiprocessor mode based on DSP and FPGA is used to combine the strapdown inertial navigation system(SINS) and global positioning system(GPS) to design the integrated navigation system for a certain type of guided aerial bomb, and design the software and hardware of the system in detail and expound some core algorithms. Through the experiment, the principle prototype designed by this scheme can meet the actual needs.
\end{abstract}

\section{Introduction}

The guided aerial bomb is a kind of air-to-ground weapon. It is equipped with a guidance system on the basis of a common missile, which has the characteristics of low cost, high precision and great function. Because of the combination of the strapdown inertial navigation system (SINS) and the global positioning system(GPS), it can be used under bad weather conditions. The short time navigation of SINS and its characteristics of high accuracy, high stability and autonomous navigation, and the continuous precise positioning of GPS for 24 hours greatly improve the accuracy and anti-jamming ability of navigation.

\section{The Overall Design of System}

\section{Requirements for Integrated Navigation System}

The first point is to complete the transfer alignment of air moving base of the inertial navigation.

The second point is to monitor the flight parameters of the guided bomb in real time.

The third point is to give the star number, ephemeris, precision factor and other status data in real time.

The fourth point is the synchronization time for the pulse of each component on the missile.

\section{The Interface Design of Integrated Navigation System}

Before dropping the bomb, the navigation system must receive a large number of navigation parameters to complete the transfer alignment of the air moving base. This process has the characteristics of large data volume and large amount of transmission and complex structure. Therefore, the interface relationship between the navigation system and subsystems of the bomb must be designed in detail. The specific design of the system interface is shown in figure 1 .

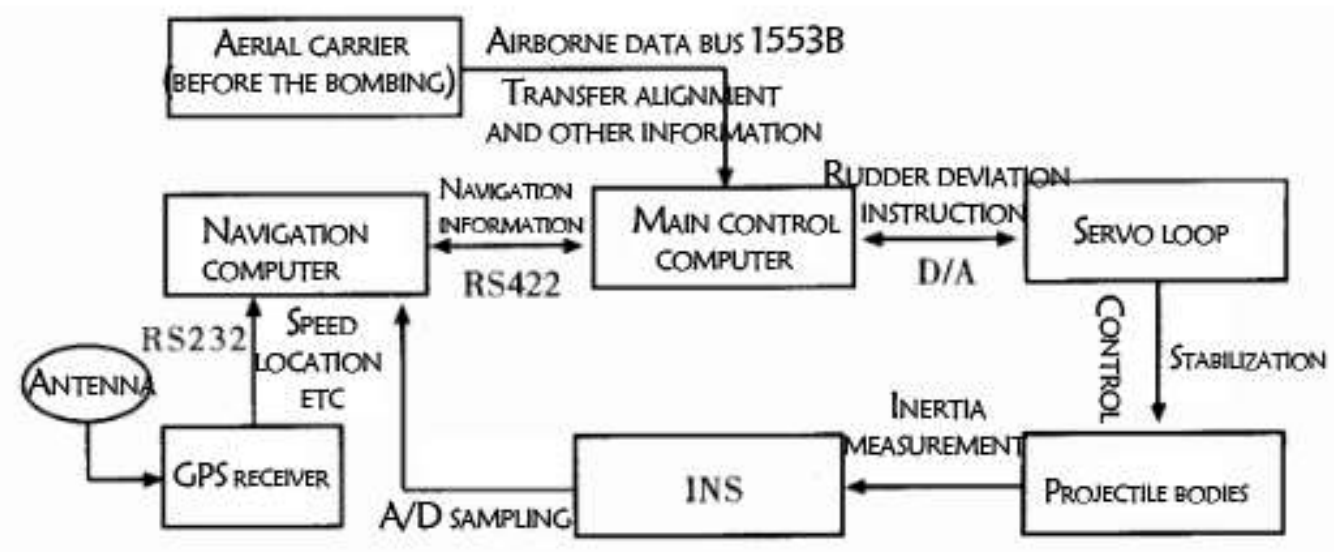

Figure 1. System interface diagram 


\section{Hardware Design}

\section{Strapdown Inertial Navigation and GPS Receiver}

The production technology of flexible gyroscopes is becoming more and more mature. The general accuracy is around $0.1 \% \mathrm{~h}$, and the price is low. A double-axis gyroscope is around $16000-18000$ yuan, so this design uses the IMU of the latest flexible gyroscope produced by a factory. The gyroscope of micro-electro-mechanical system(MEMS) can effectively reduce the cost, but the MEMS technology is not yet mature and the accuracy is low, and it can't guarantee that it can be used alone in the case of GPS failure, so in order to ensure the stability, the IMU of the flexible gyroscope is selected.

The GPS receiver uses the JNS100 double-star receiver of the JAVAD company in the United States. Its price is low and it has high dynamic positioning precision. Its real-time update rate of original data and location data is up to $10 \mathrm{HZ}$, and the recapture time is less than $1 \mathrm{~s}$, and it also provides ASCH code navigation message output and the output of binary original measurement data, which is an ideal choice for integrated navigation system.

\section{Navigation Computer Based on Floating Point DSP}

Navigation computers need to calculate a lot of information in a short time, and frequently do the work of A/D sampling, GPS data acquisition and communication with external systems, which is a great test for computer's computing speed and processing capability. Therefore, the floating point DPS+FPGA mode is selected, and DSP uses the 32-bit floating point TMS320C6713 and the main frequency is 200MIPS. Its real number operation speed can reach 18 million floating points operation per second, and the operation of eighteenth-order adaptive combination filter only needs $7 \mathrm{~ms}$, and the calculation part of inertial navigation is no more than $1 \mathrm{~ms}$, which fully meets the speed requirement. It also supports the 64-bit double precision floating-point number of the institute of electrical and electronics engineers, and the accuracy of operation can fully meet the requirements of the system[3].

\section{Other Hard ware Options}

The minimum resolution of the measuring precision of the flexible gyroscope can be $0.001 \%$, so when the gyroscope calibration factor is $45 \mathrm{mV}-55 \mathrm{mV} / \mathrm{s}$ and the $\mathrm{A} / \mathrm{D}$ voltage range is $10 \mathrm{~V}$, the $\mathrm{A} / \mathrm{D}$ digits required for the gyroscope can be accounted for more than 17.6, and the A/D digits required for the accelerometer are calculated to be more than 14. Therefore, the system adopts 24-bit ADS1256 of ADC company, which can get 23-bit effective resolution. The sampling frequency is $20 \mathrm{ksps}$, and the 8-channel multicircuit switch is integrated in the chip, so as to ensure the accuracy of data sampling. In order to reduce errors in the interaction with external information, one of two timers in the DSP chip is used as a time recorder to measure the delay time caused in the process of information transmission or operation, and then algorithm compensation is made. The other timer acts on the timing control of the system.

The system uses eight-channel digital output and eight-channel digital input signals, which can meet the basic requirements of the integrated navigation system. The whole hardware system structure is shown in figure 1.

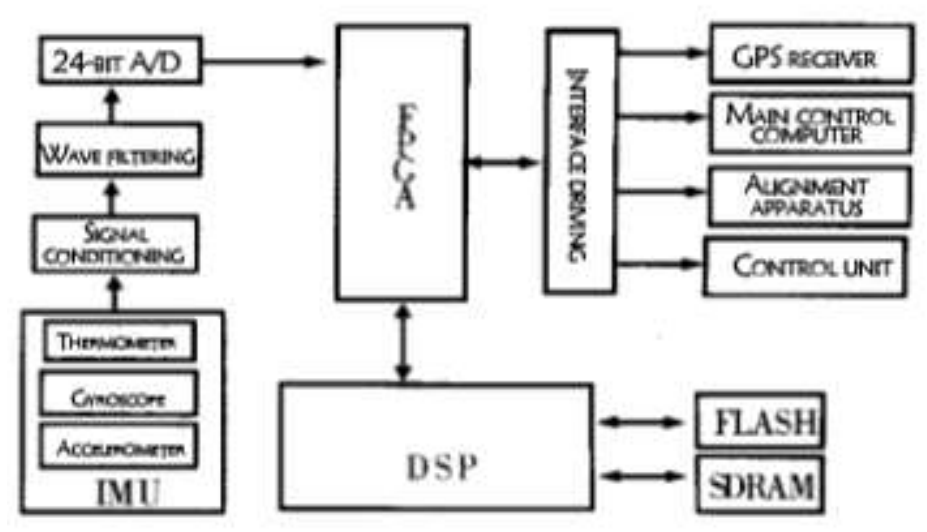

Figure 2. System hardware structure diagram 


\section{Software Design}

The software part of the integrated navigation system consists of the self-checking module of the system, the initial calibration module, the inertial navigation calculation module, the information fusion module and the monitoring and communication module. FPGA is responsible for completing the control and communication of the system, and the software is completed in the QUARTUS2.0 environment by using the VHDL language. DSP is responsible for completing the inertial navigation calculation and information fusion part, and it is implemented by C language in CCS2 environment. The core algorithm of the system is implemented by assembly program to improve the computing speed of the system. The specific functions are shown in figure 2.

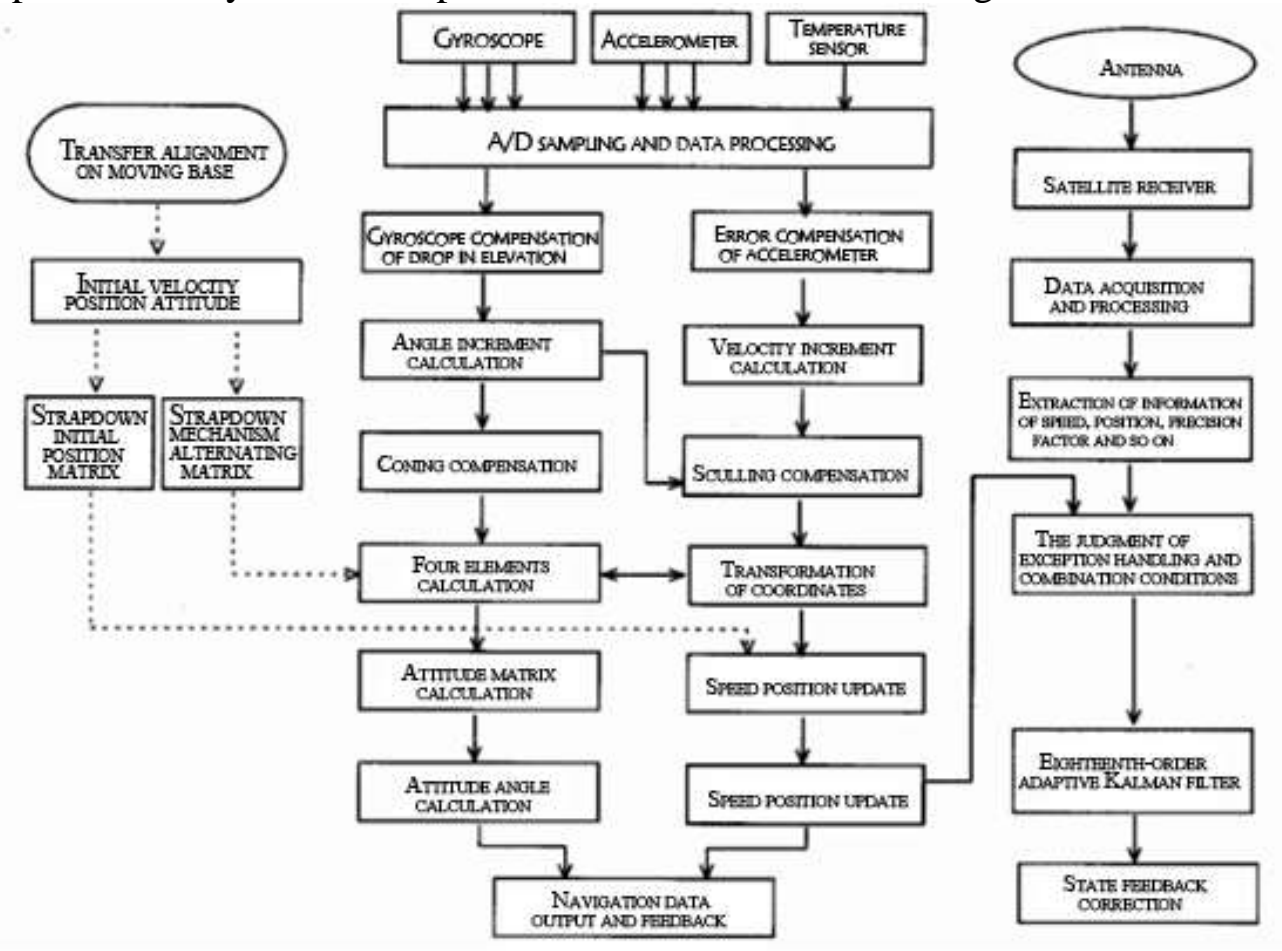

Figure 3. System algorithm flow chart

The electricity monitoring on the system is accomplished by self-checking module, covering the $\mathrm{AD}$ acquisition data detection, gyroscope working state detection, computer system detection, the correctness detection of memory reading and writing and program loading, the correctness detection of serial port communication and calibration coefficients loading, and the detection of the link of GPS system and the data validity.

The initial check module can realize the velocity matching of the transfer alignment of the moving base of the main inertial navigation and missile-borne inertial navigation of warplanes in the bombing area. The horizontal pointing accuracy is higher than $0.05^{\circ}$, and the azimuth pointing accuracy is about $0.09^{\circ}-0.12^{\circ}$. The alignment process is shown in figure 3 . 


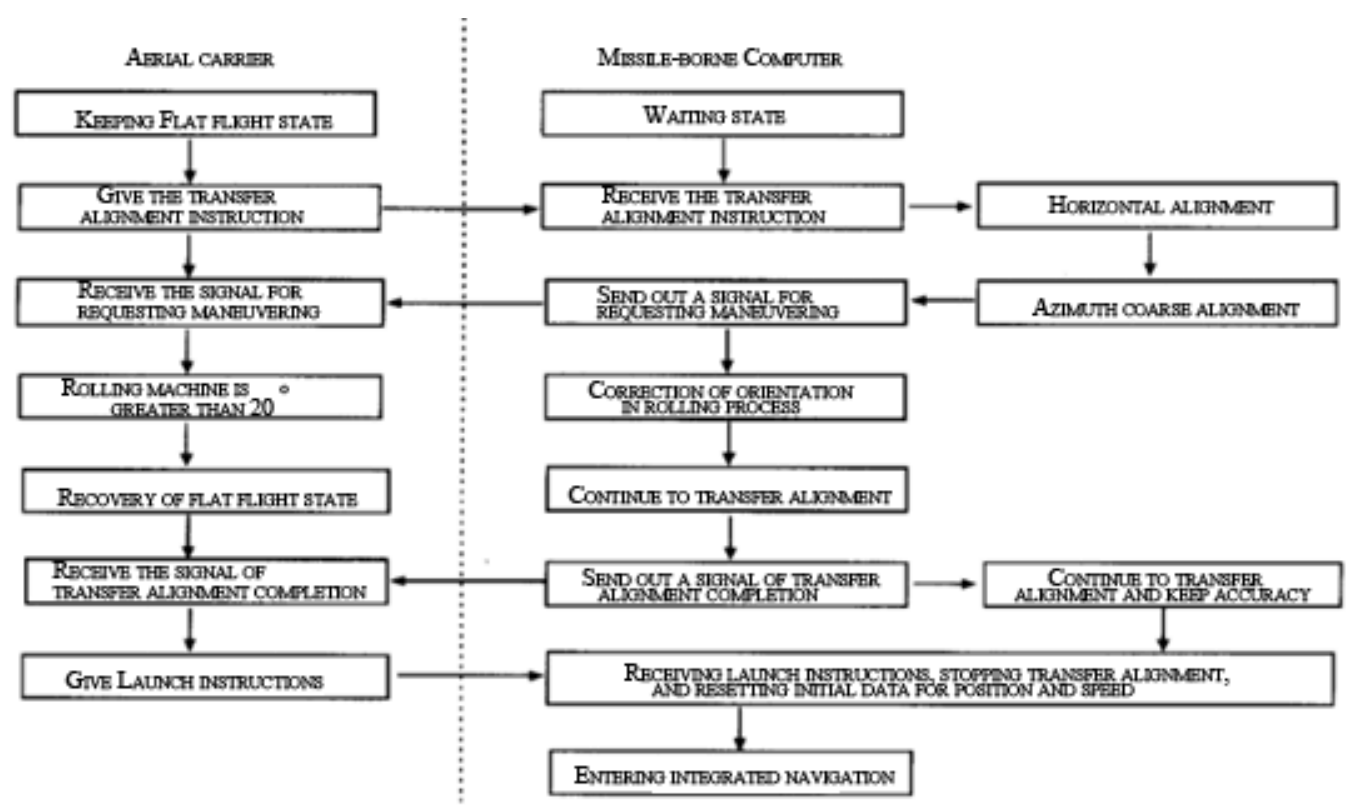

Figure 4. Transfer alignment flow chart of moving base

The calculation of the strapdown matrix, the error compensation of the inertial instrument and the calculation of the attitude matrix are completed by the inertial navigation calculation module. The computer collects the data of the gyroscopes and accelerometers every millisecond and calculates the acceleration and angular velocity every 5 milliseconds. The navigation calculation uses the quaternion update two-sample algorithm, and a frame of navigation data is output every 10 milliseconds.

The problem of continuous interruption caused by satellite switching can be effectively controlled by using eighteenth-order kalman filter. The indirect method of feedback correction is adopted, and the amplitude of the correction is limited. The limiting amplitude can be determined based on the time of correction, the magnitude of the last correction and the relative error of GPS data. The uncertain factors of the external environment and the instability of components of combined system such as gyroscope and accelerometer lead to inaccurate systematic observation noise, and the traditional kalman filter can not guarantee its stability. Therefore, whether from the point of view of the real-time performance of the algorithm or the reduction of the error, it is the right choice to adopt the fading adaptive kalman filter[2]. The fading adaptive filter algorithm is as follows:

State one step prediction equation:

Measurement correction equation:

Filter gain equation:

One step prediction mean square error equation:

Estimation mean square error equation:

The difference between the fading adaptive kalman filter and the conventional kalman filter is that the former multiplies the adaptive forgetting factor $\lambda_{\mathrm{k}}$ when calculating one step prediction

\section{$\mathbf{P}$}

mean square error

. The purpose is to use the forgetting factor to limit the memory length of kalman filter. By using the current measurement data, the role of data in state estimation can be increased and the filtering divergence can be avoided. A simple algorithm for using the best forgetting factor in the system: Among them, The monitoring module is responsible for completing the communication with the monitoring computer. The computer monitors the inertial navigation system through the RS232 serial port. The module is written and processed by using VC++6.0 language, and the relevant information of the system is displayed in real time through the dial and 
animation, such as speed, attitude, location and so on. At the same time, the IMU is calibrated systematically, and the calibration coefficient is loaded into the navigation computer[1].

\section{The Experimental Process of Integrated Navigation System}

After the design of the principle prototype, the feasibility of the integrated navigation system was tested in order to test the measuring accuracy of the system. The whole integrated navigation system and principle prototype were installed on the vehicle at the same time. According to the detailed steps of the transfer alignment flow chart of the moving base, the transfer alignment of the moving base was carried out for the principle prototype. After the completion of the transfer alignment, we selected a rectangular area, and let the car run laps repeatedly(4-6 laps). And then, we selected 8 reference points on the route of the car, recorded the time series of the car passing through the reference points, collected the output parameters of integrated navigation system in the fixed line when the car ran laps, and used the mathematical software MATLAB to deal with the experimental data. The partial processing results are shown in figure 5.

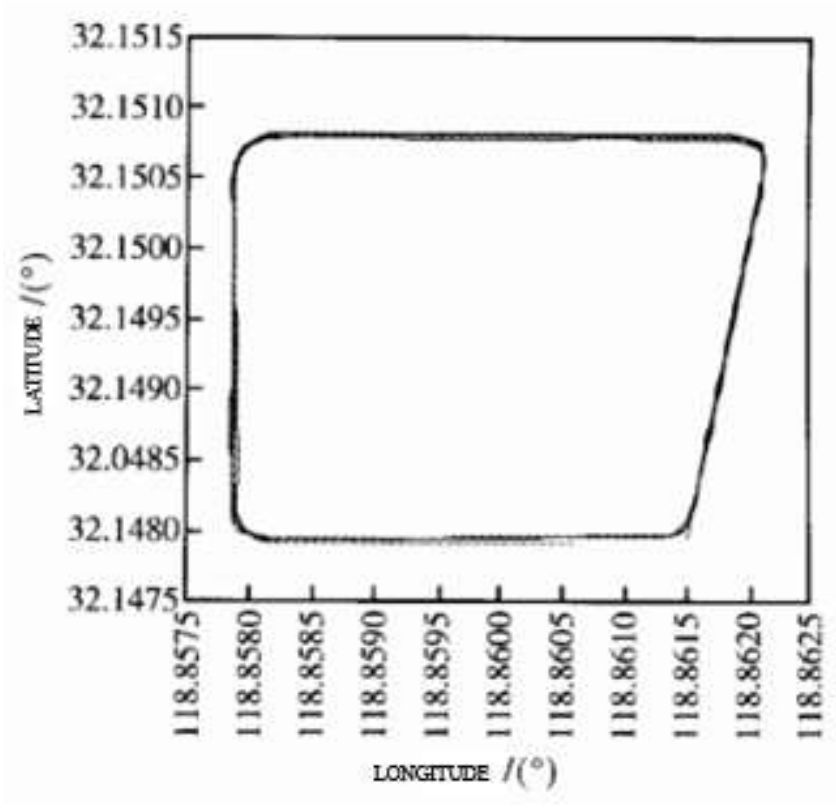

Figure 5. System experimental track chart

\section{Experimental Conclusion}

The experimental results show that the SINS/GPS integrated navigation system based on DSP+FPGA is successful, and it can accurately reflect the change of carrier speed and position in real time. The SINS/GPS integrated navigation system has the characteristics and advantages of high precision, low cost, small volume and high stability, which can meet the actual demand.

\section{Conclusion}

This paper introduces the integrated design of SINS/GPS integrated navigation system based on the DSP+FPGA in detail, and studies the SINS/GPS integrated navigation system for a certain type of guided aerial bomb. Through detailed discussion of software system design and ground test of principle prototype, the feasibility of the system is fully demonstrated. The combination of SINS/GPS can greatly improve the accuracy and anti-jamming ability of guided aerial bomb.

\section{References}

[1] Pengzhang Wang. Design and Implementation of Integrated navigation redundancy controller based on DSP+FPGA[D], Nanjing University of Science and Technology, 2017. 
[2] Yuheng Shen, Jier Qiu, Di Zhang, etc. Study on Integrated Guidance and Control Design for Side-Window Missile Based on Adaptive Sliding Modes[J], Aerospace Shanghai, 2017, 34(2):61-66.

[3] Dan Liao. Design of Hardware Platform for Navigation System by Using DSP and FPGA [J], Electronic Measurement Technology, 2017, 40(5):201-204. 